\title{
Comparison of semi-extended and standard lymph node dissection in radical prostatectomy: A single-institute experience
}

\author{
SENJI HOSHI $^{1,2}$, NATUHO HAYASHI ${ }^{1}$, YUUTA KUROTA ${ }^{1}$, KIYOTSUGU HOSHI ${ }^{2}$, AKINORI MUTO ${ }^{1}$, \\ OSAMU SUGANO ${ }^{1}$, KENJI NUMAHATA ${ }^{1}$, VLADIMIR BILIM ${ }^{3}$, ISOJI SASAGAWA ${ }^{2}$ and SHOICHIRO OHTA ${ }^{4}$ \\ ${ }^{1}$ Department of Urology, Yamagata Prefectural Central Hospital, Yamagata, Yamagata 990-2292; ${ }^{2}$ Department of Urology, \\ Yamagata Tokushukai Hospital, Yamagata, Yamagata 990-0834; ${ }^{3}$ Department of Urology, Niigata Cancer Center Hospital, \\ Niigata, Niigata 951-8566; ${ }^{4}$ Clinical Pathophysiology, Faculty of Pharmaceutical Science, \\ Josai University, Sakado, Saitama 350-0295, Japan
}

Received May 7, 2015; Accepted June 18, 2015

DOI: $10.3892 / \mathrm{mco} .2015 .601$

\begin{abstract}
Standard lymphadenectomy for prostate cancer is limited to the obturator lymph nodes (LNs), although the internal and external iliac LNs represent the primary landing zone for prostatic lymphatic drainage. We performed anatomically semi-extended pelvic lymph node dissection (PLND) to assess the incidence of LN metastasis in cases of clinically localized prostate cancer. A total of 730 consecutive patients underwent radical prostatectomy with either semi-extended PLND, comprising 6 selective fields, namely the external iliac, internal iliac and obturator LNs bilaterally, or standard LND (obturator LNs alone). A total of 131 patients undergoing semi-extended PLND were compared with 599 patients undergoing standard LND. The patients were stratified into high-risk [prostate-specific antigen (PSA) $>20 \mathrm{ng} / \mathrm{ml}$, Gleason score (GS) $\geq 8$ ], intermediate-risk (PSA 10-20 ng/ml, GS=4+3) and low-risk (PSA $<10 \mathrm{ng} / \mathrm{ml}, \mathrm{GS} \leq 3+4)$ subgroups. Following semi-extended LND, positive LNs were detected in 12/61 (20\%) of the high-risk, $1 / 30$ (3\%) of the intermediate-risk and 0/40 (0\%) of the low-risk cases. Following standard LND, positive LNs were detected in 13/182 (7\%) of the high-risk, 1/164 (0.6\%) of the intermediate-risk and 0/253 (0\%) of the low-risk cases. In high-risk patients, the detection rate of $\mathrm{LN}$ metastasis was significantly higher following extended LND compared with standard LND ( $\mathrm{P}<0.01)$. In 9 of 13 patients $(69 \%)$, metastases were identified in the internal and external iliac regions, despite negative obturator LNs. There were no significant differences regarding intraoperative and postoperative complications or blood loss in the two groups. There was no lymphocele formation in patients undergoing either standard or semi-extended LND. Extended
\end{abstract}

Correspondence to: Professor Shoichiro Ohta, Clinical Pathophysiology, Faculty of Pharmaceutical Science, Josai University, 1-1 Keyakidai, Sakado, Saitama 350-0295, Japan

E-mail: sohtajp@josai.ac.jp

Key words: standard lymph node dissection, radical prostatectomy, semi-extended lymph node dissection pelvic LND (PLND) is associated with a high rate of LN metastasis detection outside the fields of standard LND in cases with clinically localized prostate cancer. Therefore, LND including the internal and external iliac LNs should be performed in all patients with high-risk prostate cancer; however, in the low-risk group, PLND may be omitted.

\section{Introduction}

Pelvic lymph node dissection (PLND) at the time of radical prostatectomy (RP) is currently the most reliable method for detecting lymph node (LN) metastases in patients with prostate cancer (1-4). Conventionally, only the obturator LNs are included in LND for prostate cancer; such a narrow dissection range may be associated with the low number of node-positive cases. Indeed, Miyake et al (5) demonstrated that localized LND in RP was insufficient. Extended LND (ELND) has been recommended, as it increases the node-positive rate in localized prostate cancer. However, an increased risk of complications associated with ELND has also been reported $(6,7)$.

To verify whether the node-positive rate is increased by widening the dissection range and whether ELND may be safely performed on a routine basis, we conducted a comparative study between a group of patients treated with the ELND proposed by Bader et al (8), where the obturator foramen LNs were excised along with the internal and external iliac LNs, and another group treated with conventional obturator LND alone.

Standard LND for prostate cancer is limited to obturator LNs, although the internal and external LNs represent the primary landing zone for prostatic lymphatic drainage. Heidenreich et al (9) reported that, considering their data, dissection of the presacral and common iliac lymphatics does not appear to be necessary, since only 3 of 95 patients (3.1\%) had LN metastasis in that region. Heidenreich et al (9) and Zincke (10) have also demonstrated the high diagnostic accuracy of pelvic staging LND limited to the internal and external iliac and the obturator fossa LNs. We referred to LND limited to the internal and external iliac and obturator fossa LNs as 'semi-extended' LND and compared it to standard LND (obturator fossa LNs alone). 
An anatomically semi-extended PLND and a conventional limited PLND were performed, in order to assess the incidence of LN metastasis in cases of clinically localized prostate cancer and evaluate the cases with metastases to the LNs.

\section{Patients and methods}

Patient stratification by treatment. We retrospectively queried our database for patients who underwent open RP between 1988 and 2013 in Yamagata Prefectural Central Hospital (Yamagata, Japan). Ethics approval was obtained from the Institutional Review Board of the Yamagata Prefectural Central Hospital (approval no. 2013-0003). Patients who received neoadjuvant or immediate adjuvant therapy were included in the study. A total of 730 consecutive patients underwent RP. The patients were divided into two groups, the group undergoing semi-extended PLND, comprising 6 selective fields, namely the bilateral external iliac, internal iliac and obturator fields, and the group undergoing standard LND (obturator LNs alone).

Patient stratification by risk. A total of 131 patients who underwent semi-extended PLND were compared with 599 standard LND patients (Table I). The patients were stratified into high-risk [prostate-specific antigen (PSA) $>20 \mathrm{ng} / \mathrm{ml}$, Gleason score (GS) $\geq 8$ ], intermediate-risk (PSA 10-20 ng/ml, $\mathrm{GS}=4+3$ ) and low-risk (PSA $<10 \mathrm{ng} / \mathrm{ml}, \mathrm{GS} \leq 3+4)$ subgroups. The patients were followed up for a median of 40 months (range, 1-261 months).

\section{Results}

Positive LN detection by treatment and risk group. Following semi-extended LND, positive LNs were detected in $12 / 61(20 \%)$ of the high-risk, $1 / 30(3 \%)$ of the intermediate-risk and $0 / 40(0 \%)$ of the low-risk cases. Standard LND identified positive LNs in 13/182 (7\%) of the high-risk, 1/164 (0.6\%) of the intermediate-risk and $0 / 253(0 \%)$ of the low-risk cases. In the high-risk group, the detection rate of LN metastasis was significantly higher following extended LND compared with that following standard LND (Table I, P<0.01).

In 9 of 13 patients $(69 \%)$, metastases were identified in the internal and external iliac regions, despite negative obturator LNs. The range, mean and median number of harvested LNs were 0-24, 6.7 and 6, respectively, with standard LND and 0-34, 10.8 and 9, respectively, with semi-extended LND $(\mathrm{P}<0.01)$ The PSA of patients with LN metastasis ranged between 3.3 and $252.7 \mathrm{ng} / \mathrm{ml}$ (mean, 49.7 and median, $38 \mathrm{ng} / \mathrm{ml}$ ). All the patients with a single obturator LN metastasis underwent standard LND.

In the 2 intermediate-risk patients exhibiting LN metastasis, the serum PSA level (GS) was $6.7 \mathrm{ng} / \mathrm{ml}(4+3)$ and $16.1 \mathrm{ng} / \mathrm{ml}(4+3)$, respectively.

Complications and outcome. There were no significant differences regarding intraoperative and postoperative complications or blood loss between the two groups. There was no lymphocele formation in patients undergoing either standard or semi-extended LND. The Kaplan-Meier curves for the oncological outcomes of progression-free survival (PFS) and cause-specific survival (CSS) are shown in Fig. 1.
Table I. Number of positive lymph nodes (LNs) stratified by risk group and extent of LN dissection (LND).

\begin{tabular}{lcc}
\hline & \multicolumn{2}{c}{ LND type } \\
\cline { 2 - 3 } Risk & Standard & Semi-extended \\
\hline Low (\%) & $0 / 253(0.0)$ & $0 / 40(0.0)$ \\
Intermediate (\%) & $1 / 164(0.6)$ & $1 / 30(3.3)$ \\
High $^{\mathrm{a}}(\%)$ & $13 / 182(7.1)$ & $12 / 61(19.6)^{\mathrm{b}}$ \\
\hline
\end{tabular}

${ }^{a} \mathrm{LN}$ positivity rate in high-risk patients: standard vs. semi-extended LND; Fisher's exact test, $\mathrm{P}<0.05$; Chi-square, $7.771 .{ }^{b} \mathrm{LN}$ positivity rate in semi-extended LND: high-risk vs. intermediate-/low-risk patients; Fisher's exact test, $\mathrm{P}<0.001$; Chi-square, 10.181.

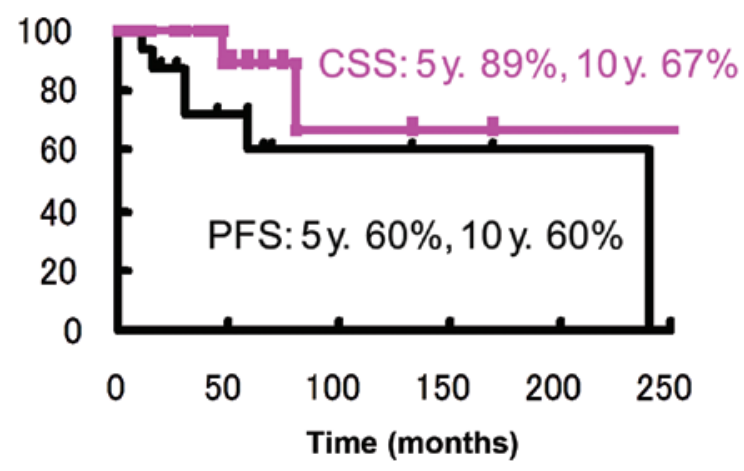

Figure 1. Kaplan-Meier curves for oncological outcomes, namely progression-free survival (PFS) and cause-specific survival (CSS) of N1 cases.

\section{Discussion}

LND in RP is considered to be of diagnostic value; however, its curative significance is limited. The detection rate of $\mathrm{LN}$ metastasis may be increased by widening the dissection range, although the extent of the dissection remains controversial. Clark et al (11) performed a prospective comparative study on extended and localized LND and observed no difference in the node-positive rate; in addition, ELND was associated with a higher complication rate. Kroepfl et al (12) included obturator and internal iliac LNs, as well as the LNs along the hypogastric vein, in the range of ELND. Heidenreich et al (9) suggested it was unnecessary to dissect the presacral and common iliac lymphatics, since only 3 of their 95 patients (3.1\%) had LN metastasis in that region. Heidenreich et al (9) and Zincke (10) demonstrated the high diagnostic accuracy of staging PLND limited to the internal and external iliac and obturator fossa LNs. An autopsy study by Weingärtner et al (13) suggested that 20 LNs must be retrieved for an adequate PLND. In the present study, the cancer-specific 5-year survival rate of pN1 patients was $89 \%$, which supports the curative significance of LND.

Retrospective analyses demonstrated that the number of positive LNs may affect survival. Certain studies have suggested that RP may be curative for a proportion of patients with LN-positive prostate cancer. Sterinberg et al (14) followed 64 patients with positive nodes, of whom 83 and $68 \%$ remained free of detectable tumor at 60 and 80 months following RP, 
respectively, and identified the tumor burden of the diseased LNs as an independent prognostic factor.

Our data suggest a delay in disease progression for prostate cancer patients with minimal LN involvement when radical surgery is performed. The follow-up duration is insufficient to reach a definitive decision in terms of possible curative effect. However, depending on the tumor burden or the number of diseased LNs, radical surgical treatment appears to improve the outcome in a not precisely defined but significant percentage of patients. Thus, meticulous pelvic LND to remove all diseased nodes and ensure accurate staging, combined with $\mathrm{RP}$, is recommended for clinically organ-confined prostate cancer. The promising survival rate of patients with minimal nodal disease makes the value of routine immediate androgen ablation in all patients with positive LNs at least questionable, particularly when considering the side effects of hormone therapy.

In conclusion, semi-extended PLND was associated with a high rate of LN metastasis detection outside the fields of standard LND in patients with clinically localized prostate cancer. LND including the internal and external iliac LNs should be performed in all patients with high risk-prostate cancer. However, in the low-risk group, PLND may be omitted.

The results of the present study demonstrated that semi-extended PLND, as performed in our institution, was associated with greater nodal yields and may result in improved oncological outcomes in patients with LN-positive prostate cancer. Although a prospective trial is required to confirm our results, we currently recommend considering semi-extended LND at the time of RP for high-risk prostate cancer patients, whereas, in the low-risk group, PLND may be omitted.

\section{References}

1. Walsh PC, Partin AW and Epstein JI: Cancer control and quality of life following anatomical radical retropubic prostatectomy: Results at 10 years. J Urol 152: 1831-1836, 1994.
2. Catalona WJ and Smith DS: Cancer recurrence and survival rates after anatomic radical retropubic prostatectomy for prostate cancer: Intermediate-term results. J Urol 160: 2428-2434, 1998.

3. Gervasi LA, Mata J, Easley JD, et al: Prognostic significance of lymph nodal metastases in prostate cancer. J Urol 142: 332-336, 1989.

4. Hull GW, Rabbani F, Abbas F, Wheeler TM, Kattan MW and Scardino PT: Cancer control with radical prostatectomy alone in 1,000 consecutive patients. J Urol 167: 528-534, 2002.

5. Miyake H, Fujimoto H, Komiyama $M$ and Fujisawa $M$ : Development of 'extended radical retropubic prostatectomy': A surgical technique for improving margin positive rates in prostate cancer. Eur J Surg Oncol 36: 281-286, 2010.

6. Withrow DR, DeGroot JM, Siemens DR and Groome PA: Therapeutic value of lymph node dissection at radical prostatectomy: A population-based case-cohort study. BJU Int 108: 209-216, 2011.

7. Schumacher MC, Burkhard FC, Thalmann GN, Fleischmann A and Studer UE: Good outcome for patients with few lymph node metastases after radical retropubic prostatectomy. Eur Urol 54: 344-352, 2008.

8. Bader P, Burkhard FC, Markwalder R and Studer UE: Disease progression and survival of patients with positive lymph nodes after radical prostatectomy. Is there a chance of cure? J Urol 169: 849-854, 2003.

9. Heidenreich A, Aus G, Bolla M, et al; European Association of Urology: EAU guidelines on prostate cancer. Eur Urol 53: 68-80, 2008.

10. Zincke H: Extended experience with surgical treatment of stage D1 adenocarcinoma of prostate. Significant influences of immediate adjuvant hormonal treatment (orchiectomy) on outcome. Urology 33 (Supp 1): S27-S36, 1989.

11. Clark T, Parekh DJ, Cookson MS, et al: Randomized prospective evaluation of extended versus limited lymph node dissection in patients with clinically localized prostate cancer. J Urol 169: 145-147, discussion 147-148, 2003.

12. Kroepfl D, Loewen H, Roggenbuck U, Musch M and Klevecka V: Disease progression and survival in patients with prostate carcinoma and positive lymph nodes after radical retropubic prostatectomy. BJU Int 97: 985-991, 2006.

13. Weingärtner K, Ramaswamy $\mathrm{A}$, Bittinger $\mathrm{A}$, Gerharz $\mathrm{EW}$, Vöge D and Riedmiller H: Anatomical basis for pelvic lymphadenectomy in prostate cancer: Results of an autopsy study and implications for the clinic. J Urol 156: 1969-1971, 1996.

14. Steinberg GD, Epstein JI, Piantadosi S and Walsh PC: Management of stage D1 adenocarcinoma of the prostate: The Johns Hopkins experience 1974 to 1987. J Urol 144: 1425-1432, 1990. 